\title{
Electroweak baryogenesis and the expansion rate of the Universe
}

\author{
Michael Joyce \\ Theory Division, CERN, 1211 Geneve 23, Switzerland
}

(Received 4 June 1996)

\begin{abstract}
The standard requirement for the production of baryons at the electroweak phase transition, that the phase transition be first order and the sphaleron bound be satisfied, is predicated on the assumption of a radiationdominated universe at that epoch. One simple alternative, domination by the energy in a kinetic mode of a scalar field which scales as $1 / a^{6}$, gives a significantly weakened sphaleron bound for the preservation of a baryon asymmetry produced at a first-order phase transition, and allows the possibility that the observed baryon asymmetry be produced when the phase transition is second order or crossover. Such a phase of "kination" at the electroweak scale can occur in various ways as a scalar field evolves after inflation in an exponential potential. [S0556-2821(97)05504-5]
\end{abstract}

PACS number(s): 98.80.Cq, 11.30.Fs, 12.15.Ji

The Hubble expansion rate $H$ of a homogeneous and isotropic Big Bang universe is given by the very simple formula

$$
H^{2}=\left(\frac{\dot{a}}{a}\right)^{2}=\frac{8 \pi G}{3} \rho-\frac{k}{a^{2}},
$$

where $a$ is the scale factor, $\rho$ is the energy density, and $k$ is a constant which depends on the spatial curvature [1]. The main contribution to $\rho$ today comes from matter which scales as $1 / a^{3}$, with perhaps also a curvature term and even a small cosmological ( $\rho=$ const) term. Going back in time the scale factor decreases and the energy density in the microwave background radiation blueshifts, scaling as $1 / a^{4}$, until it comes to dominate the right-hand side of Eq. (1). The most impressive evidence for this extrapolation comes from nucleosynthesis. The precise abundances of the various nuclei synthesized from the nucleons as the Universe cools below $\sim 1 \mathrm{MeV}$ depends sensitively on the relation between the temperature of the radiation (which goes as $1 / a$ ) and the expansion rate, and the radiation-dominated picture does remarkably well.

Going back further in time we reach the electroweak epoch at $T \sim 100 \mathrm{GeV}$. The expansion rate again enters in determining the details of the relics left behind, most notably the baryon asymmetry [2]. In this paper it is pointed out that relaxing the standard assumption of radiation domination at the electroweak scale has important consequences for electroweak baryogenesis. The fact that the sphaleron bound and the usually assumed impossibility of baryogenesis at a second-order or crossover phase transition are highly dependent on this assumption is illustrated with the example of a universe dominated by the energy in a kinetic mode of a scalar field. Other examples of alternatives to radiation domination before nucleosynthesis have been discussed in works of Barrow [3] and Kamionkowski and Turner [4], who consider how the relic abundances of dark-matter particles are changed in such scenarios.

Consider first the dynamics of a real scalar field $\phi$ with potential $V(\phi)$. Variation of the action

$$
\mathcal{S}=\int d^{4} x \sqrt{-g}\left[\frac{1}{2} g^{\mu \nu}\left(\partial_{\mu} \phi\right)^{\dagger}\left(\partial_{\nu} \phi\right)-V(\phi)\right],
$$

taking the Friedmann-Robertson-Walker (FRW) metric with scale factor $a(t)$, gives the equation of motion for the homogeneous modes, which can be written

$$
\frac{d}{d t}\left(\frac{1}{2} \dot{\phi}^{2}+V(\phi)\right)+3 H \dot{\phi}^{2}=0
$$

after multiplication by $\dot{\phi}$. Defining $\eta(t)=V(\phi) /(1 / 2) \dot{\phi}^{2}$ and writing the energy density $\rho(t)=(1 / 2) \dot{\phi}^{2}+V(\phi)$, we find

$$
\begin{aligned}
\rho(t) & =\rho\left(t_{0}\right) \exp \left(-\int_{t_{0}}^{t} \frac{6}{1+\eta(t)} H(t) d t\right) \\
& =\rho\left(t_{0}\right) \exp \left(-\int_{a_{o}}^{a} \frac{6}{1+\eta(a)} \frac{d a}{a}\right)
\end{aligned}
$$

When the kinetic energy dominates $\eta \rightarrow 0$ and

$$
\rho \propto \frac{1}{a^{6}} .
$$

This represents the opposite limit to inflation driven by the potential energy with $\eta \rightarrow \infty$ and $\rho(t) \approx \rho\left(t_{0}\right)$. Indeed for any homogeneous mode [assuming only that $V(\phi)$ is positive] we have that

$$
\rho\left(t_{0}\right)\left(\frac{a_{0}}{a}\right)^{6} \leqslant \rho(t) \leqslant \rho\left(t_{0}\right), \quad t \geqslant t_{0} .
$$

Putting these limiting behaviors of the energy density into Eq. (1) one finds $a \propto t^{1 / 3}$ (with $k=0$ ) for the $1 / a^{6}$ scaling, in contrast to $a \propto e^{H t}$ for inflation ( $H=$ const). Instead of superluminal expansion in inflation a kinetic-energy-dominated mode of a scalar potential drives a subluminal expansion very similar to that of radiation $\left(a \propto t^{1 / 2}\right)$ or matter $\left(a \propto t^{2 / 3}\right)$. Writing the stress energy tensor in terms of a pressure $p$ and the energy density in the standard way, the equa- 
tion of state is $p=\rho$ for the kinetic mode in contrast to $p=(1 / 3) \rho$ (radiation), $p=0$ (matter), and $p=-\rho$ (inflation).

I will use the term kination to refer to a phase of the Universe dominated by the kinetic energy of a scalar field. The "deflationary" universe of [5], which will be discussed below, is a particular example of this, in which the inflaton evolves into such a kinetic mode [6].

Now let us suppose that an unknown amount of energy is stored in such a mode at the electroweak epoch. The expansion rate in Eq. (1) becomes

$$
H^{2}=\left(\frac{\dot{a}}{a}\right)^{2}=\frac{8 \pi G}{3} \frac{\rho_{e}}{2}\left[\left(\frac{a_{e}}{a}\right)^{6}+f(a)\left(\frac{a_{e}}{a}\right)^{4}\right],
$$

where $a_{e}$ is the scale factor when the density in the mode becomes equal to that in radiation and $\rho_{e}$ is the energy density at that time. The factor $f(a)$ accounts for the effect of decouplings, and in the approximation that they are instantaneous is $f(a)=\left[g\left(a_{e}\right) / g(a)\right]^{1 / 3}$ where $g(a)$ is the number of relativistic degrees of freedom. The sphaleron bound [7] results from the requirement that the rate of baryon number violating (sphaleron) processes after the electroweak phase transition be less than the expansion rate of the Universe so that the baryon asymmetry (putatively) created at the electroweak phase transition be "frozen in." Thus

$$
\Gamma_{\mathrm{sph}} \sim T_{w} e^{-E_{\mathrm{sph}} / T_{w}<H_{6}}=\left(\frac{H_{6}}{H_{4}}\right) H_{4},
$$

where $H_{6}$ is the Hubble expansion rate and $H_{4}=1.66 \sqrt{g_{w}}\left(T^{2} / M_{p l}\right)$ is the expansion rate we get if we assume radiation domination in the usual way, with $g_{w}=$ $g\left(a_{w}\right) \sim 100$ and $T_{w} \sim 100 \mathrm{GeV}$. The bound on $E_{\text {sph }}$, the sphaleron energy, can thus be written in terms of the usual bound on the same quantity $E_{\mathrm{sph}}^{0}$ as

$$
E_{\mathrm{sph}}=E_{\mathrm{sph}}^{0}-T_{w} \ln \left[\left(\frac{g_{w}}{g_{e}}\right)^{1 / 2} \frac{T_{w}}{T_{e}}\right] .
$$

This follows since $H_{6} / H_{4} \approx\left[1 / \sqrt{f\left(a_{w}\right)}\right]\left(a_{e} / a_{w}\right)$ and $T a=f(a) T_{e} a_{e}$, where $T_{e}$ is the temperature at radiationkinetic energy equality (at $a=a_{e}$ ).

Let us take the following approximate bound from nucleosynthesis: We allow $10 \%$ of the energy to come from the coherent mode at $\sim 1 \mathrm{MeV}$, just before the first stage of $n-p$ freezout begins [8]. Then $T_{e} \sim 3 \mathrm{MeV}$, so taking $T_{w} \sim 100 \mathrm{GeV}$, the bound on the sphaleron energy is reduced by approximately one-quarter from its usual value of $\sim 45 T$ [2]. The lower bound on $E_{\mathrm{sph}}$ can be translated into constraints on the parameters in the zero-temperature theory, most notably an upper bound on the lightest Higgs particle. Constraints are usually derived using the bound expressed as the ratio of the vacuum expectation value (VEV) $v$ in the nucleated bubbles to the nucleation temperature $T_{b}$, to which the sphaleron energy is linearly proportional. Typically therefore the sphaleron bound will be weakened as

$$
\frac{v}{T_{b}}>1 \rightarrow \frac{v}{T_{b}}>0.75
$$

How significant a difference is this? According to recent lattice studies of the electroweak phase transition in the minimal standard model $[9,2]$, the "usual" sphaleron bound cannot be satisfied for any physical Higgs boson mass, for a top quark mass of $m_{t}=175 \mathrm{GeV}$. The "new" bound in Eq. (10) is satisfied for Higgs boson masses up to about $35 \mathrm{GeV}$. For $m_{t}=155 \mathrm{GeV}$ the bound changes from about $35 \mathrm{GeV}$ (for the "usual" case) to $50 \mathrm{GeV}$. The "new" bounds are still however too low to be consistent with the CERN $e^{+} e^{-}$collider LEP bounds on the standard model Higgs boson mass $m_{H}>65 \mathrm{GeV}$.

In extensions of the standard model, such as the minimal supersymmetric model (MSSM), recent perturbative [10] and nonperturbative [11] analyses indicate that the usual sphaleron bound can be satisfied in various parts of experimentally allowed parameter space. The new bound simply widens this allowed parameter space. In what sense can this widening be said to be significant or not? For baryogenesis what one must calculate given any set of physical parameters (ultimately to be fixed by particle physical experiments, we hope) is a depletion factor $X$, where $B_{f}=e^{-X} B\left(T_{0}\right)$ is the baryon number at nucleosynthesis and $B\left(T_{0}\right)$ is the baryon number created during the departure from equilibrium at some temperature $T_{0}$ (usually very close to the critical temperature for the phase transition). It is simple to show that

$$
X=\int_{t_{0}}^{\infty} d t \Gamma_{\mathrm{sph}}(t)=H_{0}^{-1} \int_{0}^{T_{0}} d T \frac{\Gamma_{\mathrm{sph}}}{T}\left(\frac{T_{0}}{T}\right)^{p},
$$

where $p=2$ in the case of radiation domination, and $p=3$ for kination. The extra power in the integral is negligible because the integral is cutoff very rapidly due to the exponential dependence in the sphaleron rate, so that the depletion factor is simply changed in inverse proportion to the expansion rate at the phase transition $H_{0}$. The estimate given above allowing for the potential contribution of the kinetic mode corresponds to a change in the expansion rate by up to a factor of $10^{5}$ [the factor inside the logarithm in Eq. (9)], so that it could make the difference in a given model between an asymmetry consistent with observation, and one $e^{-10^{5}}$ times smaller. This is certainly in an absolute sense a significant difference.

Has such a change to the expansion rate other consequences? An expansion rate at the electroweak scale of $\sim 10^{-11} T$, instead of $\sim 10^{-16} T$ in the radiation-dominated case, leaves the usual treatment of the phase transition intact, because the time scale for the expansion is still very long compared to thermalization time scales. Details will change. The phase transition will proceed slightly differently, e.g., with more supercooling before the nucleation of bubbles [12]. The slowest perturbative processes, those flipping the chirality of electrons which have a rate $\sim 10^{-12} T$, will remain out of equilibrium leading to minor alterations to various calculations of baryon number.

With increasing Higgs masses the phase transition becomes more weakly first order, and, according to recent nonperturbative lattice results [13] eventually (at $m_{H} \approx 80 \mathrm{GeV}$ in the standard model) the line of first-order transitions ends in a second-order transition and becomes crossover. This means that there is actually no phase transition, all gauge-invariant observables evolving continuously as a function of tempera- 
ture. In this case it has been assumed that a baryon asymmetry of the observed magnitude cannot be created, because the departure from equilibrium required by the Sakharov conditions is too small, being controlled by the expansion rate of the Universe rather than by the much shorter time scales characterizing the propagation of bubbles at a first order phase transition [14-16]. At a first-order phase transition too weak to satisfy the sphaleron bound, the same will be true as, after the completion of the phase transition, the expansion rate again becomes the relevant time scale. A very simple calculation of the baryon asymmetry is possible in these cases with the assumption of homogeneity in the evolution of the fields. In various extensions of the standard model with extra $C P$ violation there are terms in the effective action which act like chemical potentials either for baryon number $[17,18]$ or hypercharge [19]. In the presence of these source terms one finds (calculating the equilibrium with the appropriate constraints, see [20] for details) the baryon to entropy ratio

$$
\left.\frac{n_{B}}{s} \sim \frac{H_{f}}{T_{f}} \frac{1}{g_{w}} T_{f} \frac{d \theta_{C P}}{d T}\right|_{f},
$$

where $\theta_{C P}$ is the (dimensionless) $C P$-violating field during its evolution (times some model-dependent suppression) and the derivative its rate of change when the asymmetry freezes out at temperature $T_{f}$, when the expansion rate is $H_{f}$.

When the Universe is in a phase of kination, $H \propto 1 / a^{3}$ $\propto T^{3}$, so that, taking the estimate above, we can have $H_{f} / T_{f} \sim 10^{-11}\left(T_{f} / 100 \mathrm{GeV}\right)^{2}$. To evaluate the remaining factor exactly would require a full study of the detailed dynamics of the phase transition, which in this case is still well beyond current capabilities. An examination of the data available on the models studied in this regime [13] indicates that the factor multiplying $H_{f} / T_{f}$ in Eq. (12) could be as large as order one since $\Delta T$, the temperature range which characterizes the change in the quantity $\theta_{C P}$ by order one could potentially be smaller than $T_{f}$ by enough to cancel $g_{w} \sim 100$ - the transition is continuous but "sharp" (it is only because it is that it makes sense to talk of a "transition" at all). It also takes place at higher temperatures (200-300 GeV in the standard model) than when the transition is first order $\left(T_{f} \sim 100 \mathrm{GeV}\right)$. It is thus possible that an asymmetry compatible with the observed $n_{B} / s \sim(3-7)$ $\times 10^{-1}$ could result when the electroweak phase transition occurs during a phase of kination which ends just before nucleosynthesis.

The simple but important point is that the standard arguments which are used to rule out the possibility of baryogenesis at the electroweak scale in many models are predicated on the assumption of knowledge of the expansion rate. In fact the one variable in an ab initio calculation of electroweak baryogenesis which we cannot access (at least in principle) through direct measurement is the expansion rate at the electroweak epoch. Methodologically it is thus more sensible to ask what expansion rate would be required to generate the observed asymmetry in any particular model. That there is any such expansion rate is itself a very nontrivial requirement of a theory. We have just seen that allowing for the contribution of a kinetic energy dominated scalar mode opens up the possibility of the creation of the observed baryon asymmetry at a second-order or crossover phase transition. Several other possibilities have been discussed by the authors of $[3,4]$, in the context of their consideration of the dependence of the relic abundances of dark-matter particles on the expansion rate. Barrow considers the case of an anisotropic universe and Kamionkowski and Turner this and various others including a Brans-Dicke theory of gravity with the scalar dominated by its kinetic energy. In these cases the net effect is essentially described by an additional contribution to the energy density scaling as $1 / a^{6}$ just like that we have considered. Beyond these there is the possibility of other nonstandard theories of gravity such as scalartensor theories in which the gravitational constant varies. The rest of this work will concentrate on the specific model of domination by the kinetic mode of a scalar field. It is minimal in the sense that it sticks to standard Einstein gravity, and is compatible with the inflationary explanation of the homogeneity and isotropy of the observed universe.

What one requires in this case is that the energy in the kinetic mode be much greater than the energy in radiation at the electroweak scale. An explanation of the "usual" scenario in which the Universe is dominated by uniform radiation at the electroweak epoch is provided by inflation: A scalar field $\phi$ displaced from its minimum rolls in its potential $V(\phi)$, sufficiently slowly that it satisfies the condition $V(\phi) \gg \dot{\phi}^{2}$ for long enough to inflate a small uniform region outside our present horizon; the field eventually reaches its minimum and oscillates about it, until it decays to produce radiation at the "reheat" temperature $T_{\text {rh }}$. An alternative mechanism for reheating was given by Spokoiny in [5]. Instead of rolling into a minimum and oscillating, the inflaton rolls in a potential (described below) so that a period of domination by its kinetic energy follows inflation, with the resultant $1 / a^{6}$ scaling discussed above. The universe is reheated simply by particle production in the expanding universe, which is proportional to $H^{4}$ (for scalar particles nonconformally coupled to gravity). The requirement that this radiation come to dominate before nucleosynthesis requires that the transition from inflation to kination occur at a sufficiently large expansion rate, $H>10^{9} \mathrm{GeV}$. Taking the created particles to be Higgs bosons, the temperature at which thermalization occurs is estimated in [5] to be $\sim 10^{6} \mathrm{GeV}$ for the case that the transition radiation domination occurs just before nucleosynthesis. This cosmology therefore corresponds exactly to what was required in the analysis above: a universe in which there is thermalized radiation by the electroweak scale but which is dominated by a coherent kinetic mode potentially until just before nucleosynthesis.

To see that this domination by a kinetic mode over radiation can come about also in conjunction with the standard reheating scenario, we consider more carefully the sorts of potential which are required. The equations governing the dynamics of the scalar field are

$$
\begin{gathered}
\ddot{\phi}+3 H \dot{\phi}+V^{\prime}(\phi)=\frac{1}{a^{3}} \frac{d}{d t}\left(a^{3} \dot{\phi}\right)+V^{\prime}(\phi)=0, \\
H^{2}=\frac{1}{3 M_{P}^{2}}\left(\frac{1}{2} \dot{\phi}^{2}+V(\phi)\right),
\end{gathered}
$$


where $M_{P}=1 / \sqrt{8 \pi G}$ is the reduced Planck mass, and we neglect the radiation density assuming the scalar field energy to dominate. It is shown in [21] that there are particular attractor solutions to Eqs. (13) and (14) for the potential $V(\phi)=V_{0} e^{-\lambda \phi / M_{P}}$

$$
\phi(t)=M_{P} \sqrt{2 A} \ln \left(M_{P} t\right), a \propto t^{A}, \quad \frac{V(\phi)}{(1 / 2) \dot{\phi}^{2}}=3 A-1,
$$

where $\lambda=\sqrt{2 / A}$ and the origin of $\phi$ is redefined so that $V_{0}=M_{P}^{4} A(3 A-1)$. From Eq. (4) it follows that $\rho \propto 1 / a^{2 / A}$ $(\eta=3 A-1)$. Values of $A>1$ give power-law inflationary solutions and in the limit $A \rightarrow 1 / 3$, in which the kinetic energy dominates, we get the scaling associated with kination.

It is easy to see that potentials steeper than this will generically have kinetic energy dominated modes by examining the solution to Eqs. (13) and (14) with $V=0$ :

$$
\dot{\phi}(t)=\dot{\phi}_{0}\left(\frac{a_{o}}{a}\right)^{3}=\dot{\phi}_{0}\left(\frac{t_{o}}{t}\right), \quad \phi(t)=\phi_{0}+\dot{\phi}_{0} t_{0} \ln \frac{t}{t_{0}} .
$$

In any potential decreasing faster than the exponential with $A=\frac{1}{3}$ the potential terms in Eqs. (13) and (14), once smaller, will decrease faster than the other terms, and the field will approach a solution of the form (16).

Exponential potentials are particularly interesting because they occur generically in theories which are compactified, such as supergravity theories or string theories. In his analysis Spokoiny realizes the transition from inflation to kination referred to by taking an exponential potential where $\lambda$ varies in the appropriate way. If we suppose instead that the Universe goes through a period of inflation driven by some other field and reheats in the "usual" way (by oscillation and decay) leaving the radiation dominant over whatever energy density is in the exponential potential, it is simple to see [adding the contribution of the radiation to Eq. (14)] that the field begins to roll when the energies become comparable. If the exponential again has a $\lambda$ varying in the appropriate way a period of inflation which cools the radiation can occur followed by a roll of the field into a kinetic energy dominated mode as the exponential becomes steeper. Alternatively, one can consider a potential like $V_{0} e^{-\phi^{2} / M_{P}^{2}}$ with $\phi_{\mathrm{rh}} \approx 0$ (the value of the field at the end of reheating). A period of inflation (number of $e$-foldings $\sim \ln M_{P} / \phi_{\mathrm{rh}}$ ) can occur when the potential energy in the field comes to dominate. These and other models will be discussed in more detail in a forthcoming paper [20].

I am indebted to M. Shaposhnikov for many useful discussions, and to P. Elmfors, P. Ferreira, K. Kainulainen, C. Korthals-Altes, G. Moore, T. Prokopec, and N. Turok for conversations or comments. I am grateful to M. Kamionkowski for bringing my attention to [4].
[1] See, for example, P.J.E. Peebles, Principles of Physical Cosmology (Princeton University Press, Princeton, 1993); E. Kolb and M. Turner, The Early Universe (Addison Wesley, Reading, MA, 1990).

[2] For a recent review, see V.A. Rubakov and M.E. Shaposhnikov, Report No. hep-ph/9603208 (unpublished).

[3] J. D. Barrow, Nucl. Phys. B208, 501 (1982).

[4] M. Kamionkowski and M. Turner, Phys. Rev. D 37, 3406 (1988).

[5] B. Spokoiny, Phys. Lett. B315, 40 (1993).

[6] I use the term "kination" rather than "deflation" because the latter has been used elsewhere with a different meaning, cf. M. Gasperini and G. Veneziano, Mod. Phys. Lett A 8, 3701 (1993).

[7] M. E. Shaposhnikov, JETP Lett. 44, 465 (1986); Nucl. Phys. B287, 757 (1987).

[8] See, for example, B. Fields et al., Report No. astro-ph 9603009 (unpublished).

[9] K. Kajantie et al., Nucl. Phys. B456, 189 (1996).

[10] M. Carena, M. Quiros, and C. Wagner, Phys. Lett. B 380, 81 (1996); J. R. Espinosa, Nucl. Phys. B475, 273 (1996).

[11] J. Cline and K. Kainulainen, Report No. hep-ph/9605235 (un- published); M. Laine, Report No. hep-ph/9605283 (unpublished).

[12] This means that the bubbles will nucleate at a lower temperature and hence larger VEV. The sphaleron bound will thus be further weakened. This effect is, however, very small in comparison to the leading effect calculated here. See [20] for details.

[13] K. Kajantie et al., Phys. Rev. Lett. 77, 2887 (1996).

[14] V. Kuzmin, V. Rubakov, and M. Shaposhnikov, Phys. Lett. 155B, 36 (1985).

[15] For a recent discussion see T. Prokopec, R. Brandenberger, and A. Davis, Report No. hep-ph/9601327 (unpublished).

[16] One exception is a mechanism involving topological defects, cf. R. Brandenberger, A. Davis, T. Prokopec, and M. Trodden, Phys. Rev. D 53, 4257 (1996), and references therein.

[17] N. Turok and J. Zadrozny, Phys. Rev. Lett. 65, 2331 (1990).

[18] M. Dine, P. Huet, R. Singleton, and L. Susskind, Phys. Lett. B 257, 351 (1991).

[19] A. Cohen, D. Kaplan, and A. Nelson, Phys. Lett. B 263, 86 (1991).

[20] M. Joyce and T. Prokopec (in preparation).

[21] J. J. Halliwell, Phys. Lett. B 185, 341 (1987); I use the notation of J. D. Barrow, ibid. 187, 12 (1987). 